\title{
Unusual Presentation of Remethylation Disorders: A Case of Later Onset CblE Deficiency
}

\section{Silvia Kalantari ${ }^{1}$, Francesco Porta ${ }^{2 *}$, Tiziana Vaisitti $^{1}$, Silvia Deaglio ${ }^{1}$ and Marco Spada ${ }^{2}$}

${ }^{1}$ Immunogenetics and Biology of Transplantation, Città della Salute e della Scienza, University Hospital of Turin and Medical Genetics, Department of Medical Sciences, University of Turin, Turin, Italy

${ }^{2}$ Department of Pediatrics, University Hospital of Turin, Regina Margherita Children's

Hospital, Torino, Italy

*Corresponding Author: Francesco Porta, Department of Pediatrics, University

Hospital of Turin, Regina Margherita Children's Hospital, Torino, Italy.

\section{Received: October 06, 2020}

Published: December 10, 2020

(C) All rights are reserved by Francesco Porta., et al.

\begin{abstract}
Methylcobalamin deficiency type CblE is a rare autosomal recessive inborn error of intracellular cobalamin metabolism. The clinical presentation is characterized by megaloblastic anemia, delayed psychomotor development, hypotonia, seizures, nystagmus, impaired visual acuity, and failure to thrive. The symptoms onset is usually within the 3rd year of life, with most children presenting within the first. The biochemical markers of CblE deficiency are hyperhomocysteinemia, homocystinuria and hypomethioninemia. We report a case of late onset (11 yo) methylcobalamin deficiency type CblE presenting with isolated macrocytic anemia and no neurological impairment. Genetic analysis of the MTRR gene allowed to identify two compound heterozygous variants, c.626C>G; [p.(Ser209*)] and c.1715C>T [p.(Pro572Leu)], which were never reported in the literature before. The therapy with hydroxocobalamin was effective on the patient anemia and allowed to reach control of the clinical picture. The aim of this article is to show that isolated macrocytic anemia and hyperhomocysteinemia may be caused by a remethylation disorder, even when the clinical presentation is milder than expected.
\end{abstract}

Keywords: Vitamin B12 Intracellular Metabolism; CblE Disease; Late Onset; Hyperhomocysteinemia; Macrocytic Anemia

\section{Background}

Methylcobalamin deficiency type CblE (OMIM \#236270) is an autosomal recessive disease due to a defect in the intracellular metabolism of cobalamin (vitamin B12). Pathogenic variants in the MTRR gene (OMIM * 602568) and defective function of the enzyme resulting from transcription of the gene (i.e. methionine synthase reductase) are responsible for defective intracellular synthesis of methylcobalamin, the cofactor for the remethylation of homocysteine (Hcy) to methionine (Met) catalyzed by methionine synthase
(MTR gene, OMIM *156570). The enzyme methionine synthase reductase is necessary for the reaction as it maintains the enzyme methionine synthase in its functional reduced form. A defective function of the MTR gene results in methylcobalamin deficiency type CblG (OMIM \# 250940), overlapping CblE deficiency in terms of biochemical and clinical outcome. The clinical presentation of both disorders is characterized by megaloblastic anemia, delayed psychomotor development, hypotonia, seizures, nystagmus, impaired visual acuity, and failure to thrive. At variance with other 
inborn errors of cobalamin metabolism, microangiopathy and thromboembolic events, are not characteristic, although anecdotally reported [1]. Typical biochemical markers of CblE deficiency are hyperhomocysteinemia, homocystinuria and hypomethioninemia [2]. The purpose of this report is to contribute to a better characterization of the variable phenotypic spectrum associated with the disease. We show that later than expected onset of isolated macrocytic anemia together with characteristic laboratory abnormalities can be associated to a remethylation disorder, even in the absence of typical neurological abnormalities.

\section{Case Presentation}

The patient is a 20-year-old male born from healthy nonconsanguineous Caucasian parents (See figure 1 for the pedigree). He was referred to our hospital at 11 years of age because of macrocytic hemolytic anemia and severe hyperhomocysteinemia (100 $\mu \mathrm{mol} / \mathrm{L})$. Psychomotor development was normal. Treatment with intramuscular hydroxocobalamin chloride (1000 U every 3 weeks) and oral calcium folinate (15 mg twice/week) was immediately started. The macrocytosis progressively diminished and the hemoglobin levels readily normalized in spite of persistency of hyperhomocysteinemia $(80 \mu \mathrm{mol} / \mathrm{L})$. At 12 years of age the patient developed myopia and corrective lenses were needed. At 13 years of age a growth delay was apparent and the patient began a followup in the endocrinology department and reached a final height of $169 \mathrm{~cm}$, slightly lower than expected compared to parental target height. A spinal X-ray when the patient was 16 showed no skeletal anomalies. Over the years hemoglobin levels were stable (14 g/ $\mathrm{dL}$ ), macrocytosis was difficult to control (MCV = $100 \mathrm{fl}$ ) and vitamin B12 (2000 - $4000 \mathrm{pg} / \mathrm{ml}$ ) and folic acid levels (> $20 \mathrm{ng} / \mathrm{ml}$ ) were constantly high. A sovra-aortic trunk echo-color-doppler displayed no atheromatous lesion when the patient was 19 years old. No thromboembolic events were observed. Currently (20 years of age), treatment is intramuscular hydroxocobalamin chloride 5000 $\mu \mathrm{g}$ twice/week and oral calcium folinate $15 \mathrm{mg}$ twice/week. At the same age, DNA testing was performed. The clinical suspicion was focused on genes responsible for intracellular defects of vitamin B12 metabolism. Clinical exome next-generation sequencing was carried out focusing on the genes MMADHC, MTRR, MTR and $M T H F D 1$. Two heterozygous variants in different exons of the MTRR

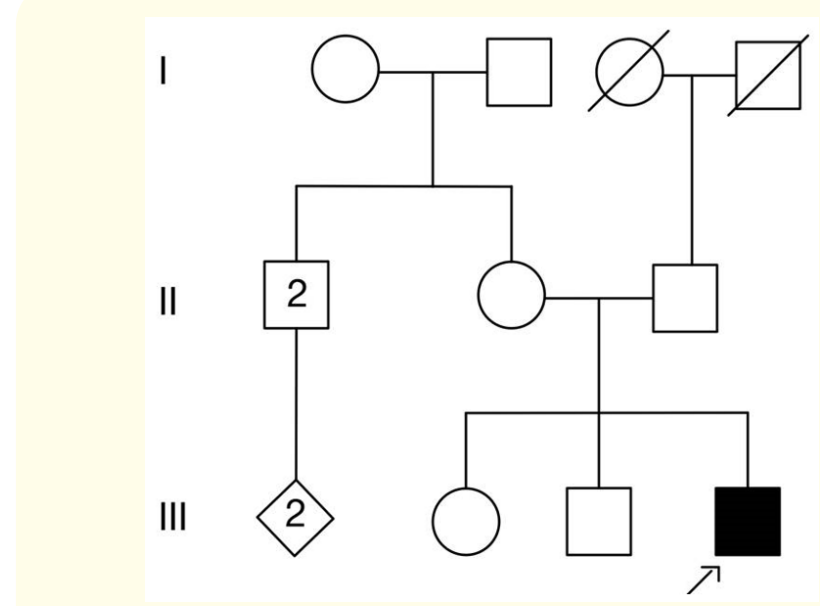

Figure 1: The patient was born from two healthy nonconsanguineous parents. He has two healthy older siblings and there were no cases of macrocytic anemia within the family.

gene were found: a likely pathogenic ( $\mathrm{C} 4$ according to ACMG guidelines [3]) truncating variant in exon 5 [c.626C>G; p.(Ser209*)] and a missense variant of unknown significance (C3 according to ACMG guidelines [3]) in exon 12 [c.1715C>T; p.(Pro572Leu)]. The presence of the two variants in compound heterozygosity (according to the family segregation of the variants) was likely responsible for the clinical presentation of the patient. The two variants have not been published in the medical literature to the authors' knowledge.

\section{Discussion and Conclusion}

Cobalamin E disease symptoms onset occurs typically before the age of 3 years, with a peak in the first year of life, when most of the patients are already symptomatic $[2,4]$. The characteristic clinical pattern encompasses feeding difficulties, hypotonia, neurocognitive impairment, failure to thrive, macrocytic anemia and eye anomalies (impaired visual acuity, nystagmus/abnormal eye movements and strabismus). Brain abnormalities at MRI are also common and encompass brain atrophy and white matter disease [2]. Microangiopathy and thromboembolism, which are frequently reported for other Cbl-related disorders, has been rarely described in CblE patients, with hemolytic uremic syndrome being a very 
rare presentation $[1,2]$. Here, we presented a clinical case of CblE disease with later-onset symptoms: the patient was brought to the attention of the hospital at 11 years of age due to a macrocytic anemia and had no history of neurocognitive impairment. Due to the very severe hyperhomocysteinemia and the absence of methylmalonic aciduria, as well as the lack of environmental causes (i.e. deficient intake of vitamin B12 with the diet) that could explain the symptoms and biochemical profile, a cobalamin-related remethylation disorder was suspected and therapy with intramuscular hydroxocobalamin chloride and oral calcium folinate was started according to current guidelines [5]. During the 9-year follow-up the patient had a decrease of visual acuity, needing corrective lenses and showed a mild growth delay, while he had a good response to the therapy, with disappearance of the anemia (hemoglobin $>14$ $\mathrm{mg} / \mathrm{dl}$, hcy $72.4 \mu \mathrm{mol} / \mathrm{L}$, plasma Met $23 \mu \mathrm{mol} / \mathrm{L})$. Three cases of very mild presentation of CblE deficiency, characterized by isolated macrocytic anemia, have already been described in the literature $[2,6]$, but in all cases the clinical presentation of the disease was far earlier than in our patient ( 2 months in one case, 3 years in the other two). Despite mild presentation of the disease, the clinical suspicion was focused when asking for the molecular genetic testing. In accordance with diagnostic algorithms proposed elsewhere $[4,5]$, once the symptoms where evocative of a Cbl-related disorder, plasma total homocysteine, plasma methionine and urinary methylmalonic acid were requested and, based on the hyperhomocysteinemia and absence of methylmalonic aciduria, the differential diagnosis lied within the range of the remethylation disorders CblD-Hcy, CblE, CblG, MTHFR deficiency or MTHFD1 deficiency. MTHFR deficiency could be excluded on clinical grounds: it is the only remethylation defect not associated with a folate trap and has no macrocytic anemia as a possible symptom [4]. MTHFD1 deficiency is a relatively newly described defect of the cytoplasmic trifunctional enzyme MTHFD1, belonging to the folate metabolism. Its presentation is indeed characterized with megaloblastic anemia and hyperhomocysteinemia and it was therefore included in the list of the possible differential diagnosis, despite the fact that immunodeficiency (lacking in our patient) is one of the typical presenting clinical features of the disorder [7]. A clear diagnostic suspicion (genes MMADHC; MTRR; MTR; MTHFD1) allowed for the laboratory in charge of the molecular testing to have a short turnaround time and to avoid unnecessary incidental findings. The two variants found in the MTRR gene were compound heterozygous and could account for the clinical picture. Isolated cobalamin-related remethylation defects, and CblE disease have a wide range of clinical presentations and no clear genotype-phenotype correlation has been established $[2,4]$. The two identified variants found in our case were never published before to the authors knowledge, and a functional validation (e.g. fibroblast cell culture and transcript analysis) would be needed. The first variant (S209X) is a truncating variant and its presence therefore likely affects the function of the protein. The second variant (P572L) is a missense substitution of uncertain significance. The in silico predictive algorithms SIFT, PolyPhen-2 and Mutation Taster were all in favor of pathogenicity and the involved amino acid is conserved (PhyloP, PhastCons). A functional validation would nonetheless be needed and will be done in the near future. In conclusion, CblE-type homocystinuria may be associated with isolated macrocytic anemia with late childhood onset. The presence of macrocytic anemia and hyperhomocysteinemia should therefore always evoke the possibility of a remethylation disorder, even when the clinical presentation is milder than expected.

\section{Conflict of Interest}

The authors declare no financial or other conflict of interest in relation to this research or its publication.

\section{Bibliography}

1. D Palanca., et al. "CblE-Type Homocystinuria Presenting with Features of Haemolytic-Uremic Syndrome in the Newborn Period". JIMD Reports 8 (2012): 57-62.

2. M Huemer., et al. "Clinical onset and course, response to treatment and outcome in 24 patients with the CblE or CblG remethylation defect complemented by genetic and in vitro enzyme study data". Journal of Inherited Metabolic Disease 38. 5 (2015): 957-967.

3. S Richards., et al. "Standards and guidelines for the interpretation of sequence variants: A joint consensus recommendation of the American College of Medical Genetics and Genomics and the Association for Molecular Pathology". Genetics in Medicine 17.5 (2015): 405-424. 
4. M Huemer and MR Baumgartner. "The clinical presentation of cobalamin-related disorders: From acquired deficiencies to inborn errors of absorption and intracellular pathways". Journal of Inherited Metabolic Disease 42.4 (2019): 686-705.

5. M Huemer., et al. "Guidelines for diagnosis and management of the cobalamin-related remethylation disorders $\mathrm{CblC}$, CblD, CblE, CblF, CblG, CblJ and MTHFR deficiency". Journal of Inherited Metabolic Disease 40.1 (2017): 21-48.

6. M A Vilaseca., et al. "CblE type of homocystinuria: Mild clinical phenotype in two patients homozygous for a novel mutation in the MTRR gene". Journal of Inherited Metabolic Disease 26. 4 (2003): 361-369.

7. P Burda., et al. "Characterization and review of MTHFD1 deficiency: four new patients, cellular delineation and response to folic and folinic acid treatment". Journal of Inherited Metabolic Disease 38. 5 (2015): 863-872.

\section{Assets from publication with us}

- Prompt Acknowledgement after receiving the article

- Thorough Double blinded peer review

- Rapid Publication

- Issue of Publication Certificate

- High visibility of your Published work

Website: www.actascientific.com/

Submit Article: www.actascientific.com/submission.php

Email us: editor@actascientific.com

Contact us: +919182824667 